\title{
Smart City, Sustainable Mobility, Home-Work Mobility:Data Analysis and Actions
}

\author{
S. Vergura \\ Department of Electrical and information Engineering \\ Technical University of Bari \\ st. E. Orabona, 4, 70125, Bari, ITALY \\ Phone:+0039 080 5963590, e-mail: silvano.vergura@poliba.it
}

\begin{abstract}
The paper deals with the issue related to the homework mobility of the employees. This issue is very important because many employees have similar working hours, starting at the same hour and finishing at the same hour. The result is a significant increase of traffic congestion and pollution in these hours. Similar situation occurs for students who have to go to school or go home. The previous cases coexist very often and are related each other. The aim of this paper is to present the results of a survey about the home-work mobility of the municipality employees of three medium cities located in the South of Italy. It results that the car is the privileged vehicle for the home-work transfer, independently from the costs of the transfer and from the time needed to reach the nearest stop of public transport vehicle. In a relevant number of cases the car is also the essential vehicle, because of intermediate mandatory stops or to the need to limit the transfer time. In these cases, the use of an electrical car could be an effective solution to join the need to use a car with the need to limit the pollution.
\end{abstract}

\section{Keywords}

Smart City, Sustainable mobility, Home-work mobility, Electrical vehicles

\section{Introduction}

Mobility in urban areas is strongly constrained, i.e., by traffic and inefficient (private and/or public) transportation. These inefficiencies lead to congestion, pollution, noise, increased energy consumption, and the associated economic losses. For instance, in the USA, congestion wastes a massive amount of time, fuel, and money. i.e., 1.9 billion gallons of wasted fuel, 4.8 billion hours of extra time, and 101 billion dollars of delay and fuel cost [1]. In the EU, congestion costs $1 \%$ of the total GDP annually [2]. According to recent studies [3], [4], the transportation sector alone accounts for $25 \%$ and $32 \%$ of the total carbon dioxide $\left(\mathrm{CO}_{2}\right)$ emissions in Europe and in the USA, respectively. Virtually all of the energy consumed in this end-use sector came from petroleum products [4]. More specifically, in the EU road transportation is responsible for $71 \%$ of the total transport share in total emissions as stated in [5] and has clearly represented the dominant mode of transport over the past few decades. One aggravating factor is the ever-increasing traffic levels that have neutralized the average emission reduction per vehicle obtained due to the design of more efficient vehicles [6]. Most of times the home-work mobility represents a criticism from the urban traffic point of view. Then we carried out a study about the homework mobility of the employees of the municipalities of three medium cities located in the South of Italy: Barletta, Andria, Trani. This study is constituted by two parts for each city. The former regards the data analysis of the questionnaire completed by the employees; the latter reports possible actions to change the standard habits of the home-work mobility in order to use less polluting transportations.

\section{Data analysis and proposed actions}

The questionnaire was administered to the municipal employees of the three cities of the province BAT (Barletta, Andria, Trani) and consists of the following main sections:

1. Report and working hours.

2. Journeys to and from work, with particular attention to the case where the user uses the car.

3. Means of transport preferred as alternative to the one already used.

The purpose of the questionnaire is to create a database of current modes of travel to and from work, of any critical situation that favors one mode (eg. the private car) over another (eg. public bus), of possible actions that could change the current choices, etc.

Fig. 1 shows, for the three municipalities involved, the total number of employees and the number of employees available to respond to the questionnaire. For the City of Barletta participated 195 employees of 292 (67\%), for the City of Andria 192 employees of 416 (46\%), for the city of Trani 86 employees of 135 (64\%).

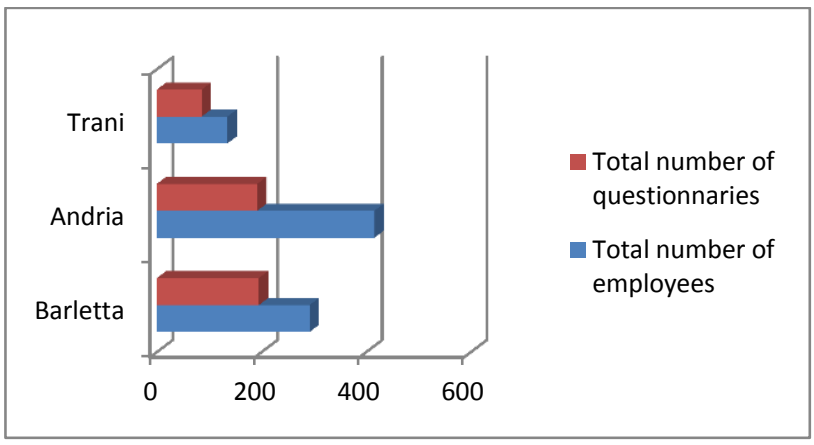

Fig. 1. Number of employees, survey participants. 


\section{A. Municipality of Barletta}

The number of municipal employees totalled 292, of which 97 (33\%) was not available to respond to the questionnaire. Of the remaining 195 questionnaires $(67 \%$ of total) 14 were partially empty, 173 employees are residents of Barletta (representing approximately $90 \%$ of the dataset available).

The first analysis concerns the mode of travel from home to work. Fig. 2 shows the number of employees for each type, which shows that the private car as driver is the main means of transport, followed by mode afoot. Very low is the use of public transport, especially the train (typically used by staff outside-seat), being very low number of employees off-seat. Compared to the other two cities, in Barletta there are also employees who use bikes/motorcycles, type virtually absent among the employees of the other two municipalities. The use of the private car (driver + passenger) represents about $49 \%$ of the mode of travel.

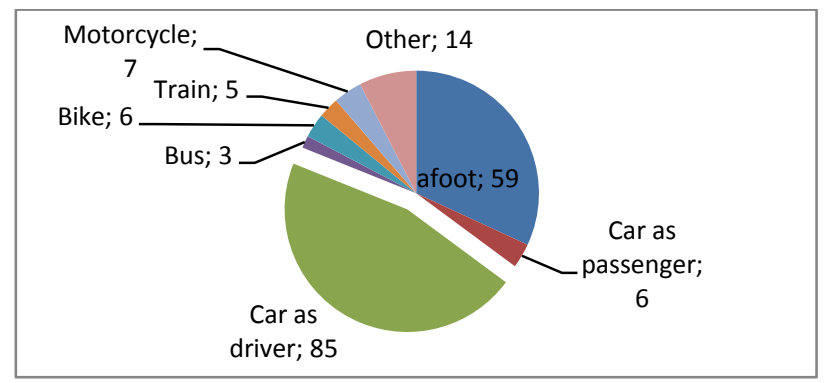

Fig. 2. Modes of travel to and from work for employees of the City of Barletta

This probably also takes account of the fact that as many as 64 employees (of the 85 ) have a private parking space in their house, which, in addition to being a head start from home, avoids the parking problem to return from work. Furthermore, over $80 \%$ of employees declared to take advantage of free parking, when it reaches the workplace, $10 \%$ use the car park of the place of work or paid parking on public land. The sum of all the values do not reach the number of questionnaires, as some employees have not given any indication of the means of transport. This situation will also occur for other times.

Another analysis concerned the time needed to reach the bus transportation mode closest to own home. Fig. 3 shows the time needed to reach the nearest bus stop, taking into account the mobility mode currently used.

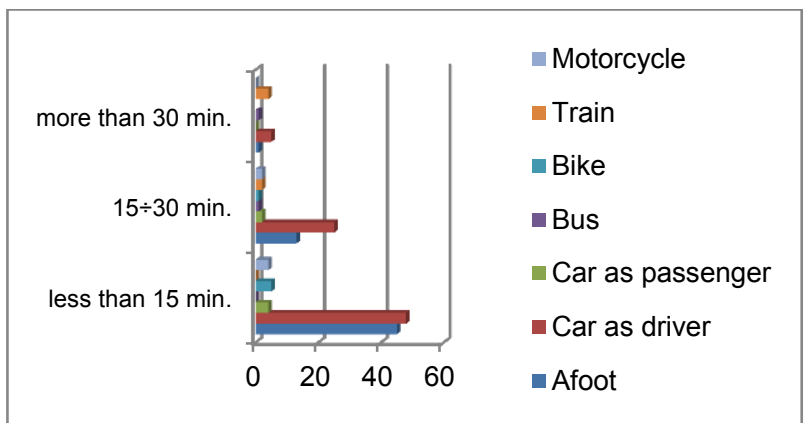

Fig. 3. Time to reach the stop nearest to your home for the employees of the City of Barletta
For times less than 15 minutes, the car as driver is the first option for the journey from home to work, followed by mode afoot. These two modes of travel account for about $95 \%$ of the mode of travel. Exceeded the 15 minutes needed to reach the nearest stop of an alternative means, the mode car as driver is the most widely used, by covering only $50 \%$ of the sample, followed by mode afoot with about $20 \%$. The set of alternative means covers about $10 \%$ of the options chosen by the employees. Some employees do not respond.

Similarly, it appears that the income of the employee does not influence the decision on the means of transport to and from work; in fact the private car is mainly used by employees, whatever the income range of belonging, thus showing a low correlation between their income and the means used (Fig. 4).

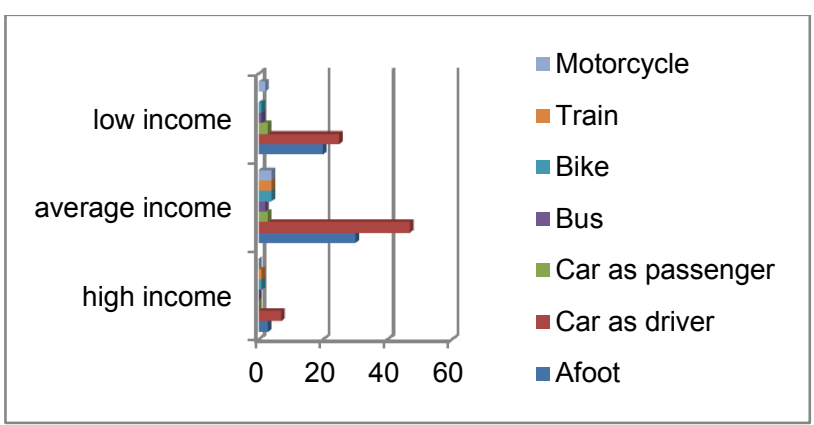

Fig. 4. Income of the employees of the City of Barletta according to the method chosen for the home-work

Finally, only 37 out of 195 state employees have to do shifts in the intermediate and from work, typically to accompany (or resume) children in sole or assistance to elderly or disabled parents or commissions. Almost all of the 37 employees use their car, even if they do occasionally intermediate displacements. Whereas the private car is used by 91 employees, this implies that about 50 employees use this medium to the only way to work, regardless of the distance and the time required to travel the distance. The fact that 8 (of 195) employees use the car as a passenger shows that less than $4 \%$ of employees use the car-pooling for commuting. Another analysis in the economic aspect is directly related to displacement. Fig. 5 shows the distribution of employees according to the monthly cost incurred for moving. $40 \%$ of employees bears a cost of less than $50 €, 46 \%$ spend between $€ 50$ and $€ 100$, the remaining $14 \%$ at a cost of more than $€ 100 /$ month and some of these more than 150 $€ /$ month.

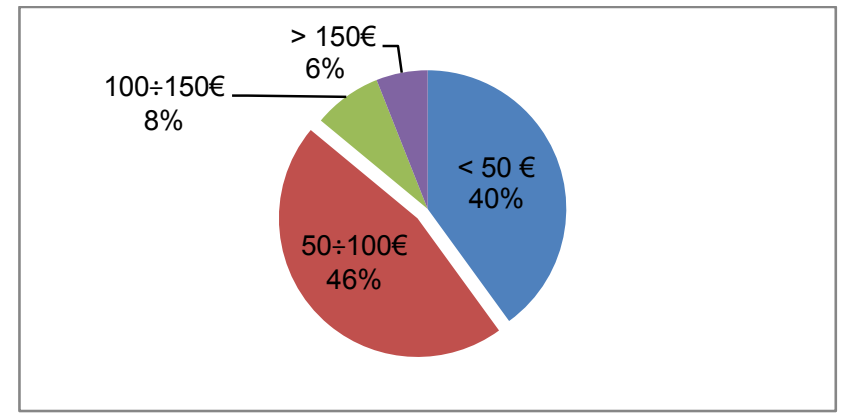

Fig. 5. Monthly cost incurred by the employees of the City of Barletta for the home-work 
Exploding the graph of Fig. 5, Fig. 6 shows that the mode of transportation car as passenger and motorcycle are the only ones with lower costs contained in $100 € /$ month. This graph, however, should be read together with that of the previous Fig. 3, which shows that the number of employees who use their own car is a substantial majority, then remedial work on this method is more effective than the other.

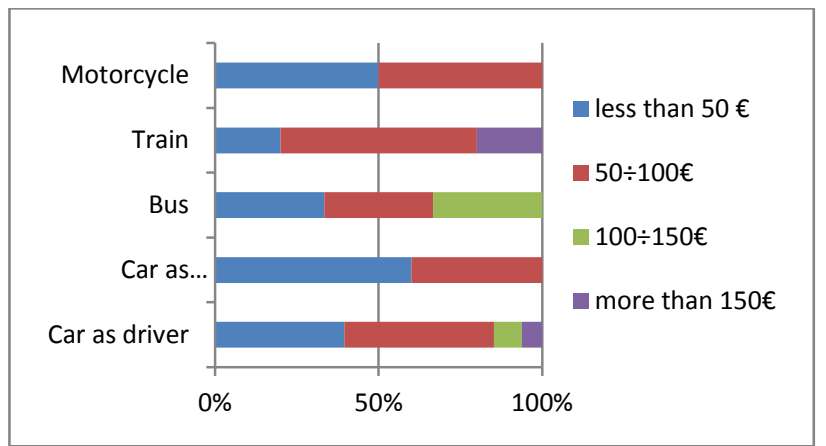

Fig. 6. Distribution of transport modes as a function of the costs incurred by the employees of the City of Barletta

As the private car is the means of transport most widely used by the employees, we have analysed the reasons for this choice. For $86 \%$ of the employees, the motivation lies in the aspects that affect the need to minimize the time spent traveling to and from work (excessive travel time, lack of direct connection, intermittent connections); only $4 \%$ believed that the use of public transport is not an economic alternative.

Given the previous result, it is asked to employees under what conditions they would be willing to use public transport. The results (Fig. 7), highlight the critical issues related to the ability to reach the workplace in certain times $(10 \%)$ and short $(18 \%)$ and at convenient times $(40 \%) .22 \%$ of employees would not use anyway public transport; for some of them the choice is linked to other needs that are not compatible with the public transportation.

Options other than public transport (Fig. 8) have been also evaluated. Car-pooling (already used by some employees) is not considered as a possible alternative by $45 \%$ of employees who use the car, while $31 \%$ would consider it, if it would not result in a time dilation and $8 \%$ if there were reserved parking space.

The bike-sharing is considered as a possible alternative by $56 \%$ of employees self-equipped (Fig. 9), provided that there are stalls equipped $(25 \%)$, best and safe bike paths $(23 \%)$ and public bike rental (8\%). The remaining $44 \%$ does not evaluate this possibility in any case.

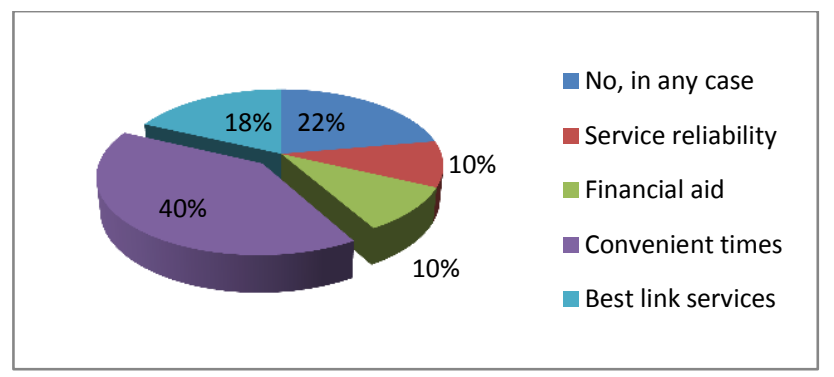

Fig. 7. Conditions of the employees of the City of Barletta to use public transport

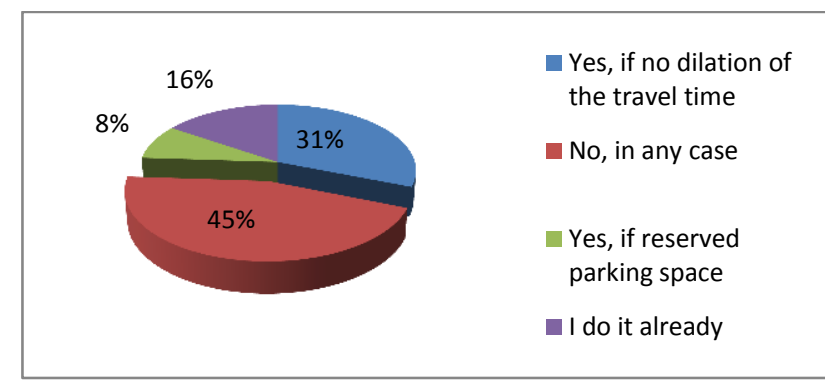

Fig. 8. Conditions of the employees of the City of Barletta to use the car-pooling

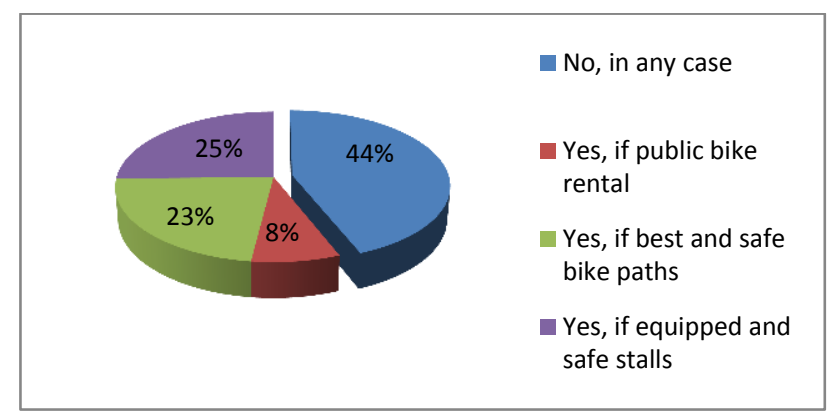

Fig. 9. Conditions of the employees of the City of Barletta to use the bike-sharing

\section{B. Municipality of Andria}

The number of municipality employees totaled 416 , of which $224(54 \%)$ was not available to respond to the questionnaire. The completed questionnaires were 192 (46\%), of which 173 employees resident in Andria.

The first analysis concerns the mode of travel from home to work. Fig. 10 shows the number of employees for each type, which shows that the private car as driver is the main means of transport, followed by mode afoot. Very low is the use of public transport, especially the train (typically used by staff outside-seat), being very low number of employees off-seat. The use of the private car (driver + passenger) represents about $56 \%$ of the mode of travel. This probably also takes account of the fact that 71 employees (90 with own car) have a private parking space in their house, which, in addition to being a head start from home, avoid the parking problem to return from work. Furthermore, all employees say they benefit from free parking, when they reach the workplace.

Another analysis concerned the time needed to reach the bus transportation mode closest to own home. Fig. 11 shows the time needed to reach the nearest bus stop, taking into account the mobility mode currently used.

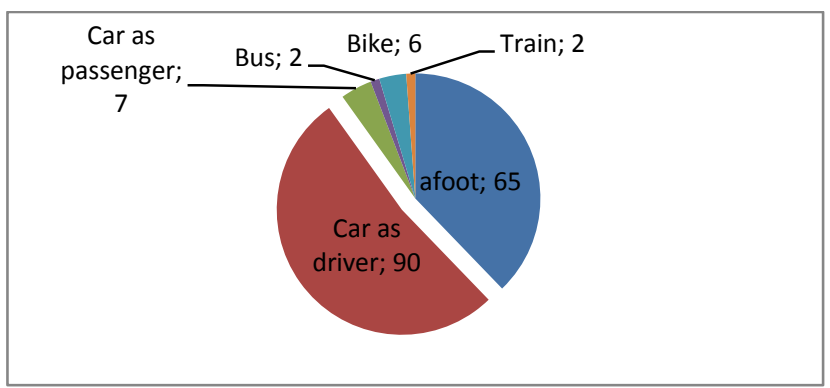

Fig. 10. Modes of travel to and from work for employees of the City of Andria 


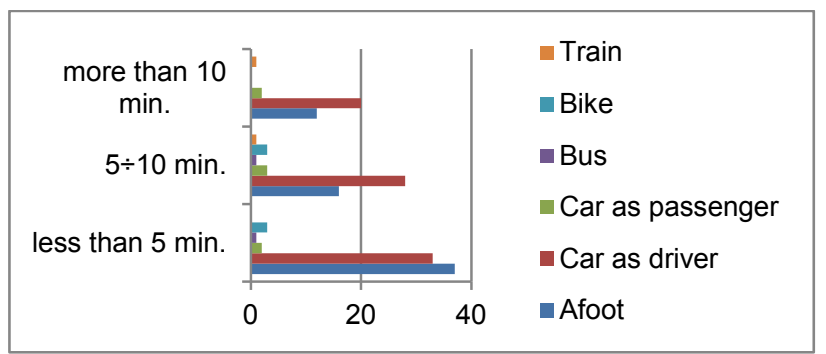

Fig. 11. Time to reach the stop nearest to your home for the employees of the City of Andria

It turns out that only time less than 5 minutes, the mode afoot is the first option for the journey from home to work, hounded by his car mode. Once 5 minutes needed to reach the nearest stop of an alternative means, the mode car as driver is the most widely used. Even the bicycle is used to route short and medium. The set of alternative means covers about $10 \%$ of the options chosen by the employees. Similarly, it appears that the income of the employee does not influence the decision on the means of transport to and from work. In fact, the private car is majority used (except mode afoot that does not have direct costs) by employees with average income as well as by employees with low income, thus showing a low correlation between their income and the means employed (Fig. 12).

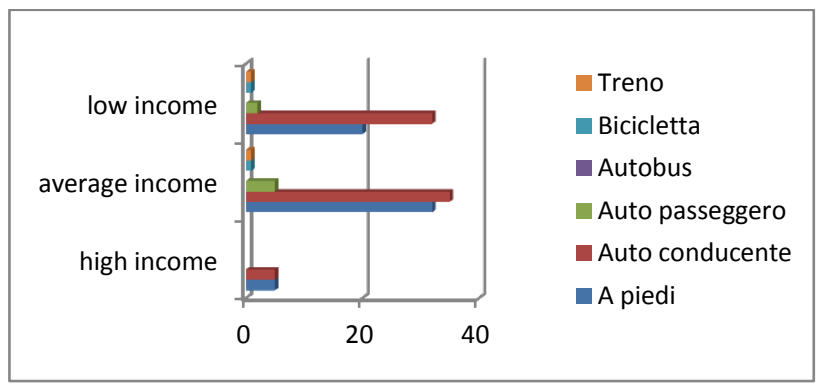

Fig. 12. Income of the employees of the City of Andria in function of your choices in the home-work

Finally, only 35 out of 192 state employees have to do shifts in the intermediate and from work, typically to accompany (or resume) children in sole or assistance to elderly or disabled parents or commissions. All 35 employees use your own car. Whereas the private car is used by 90 employees, this implies that 65 employees (plus 8 as passengers) use this medium to the only way to work, regardless of the distance and the time required to travel the distance. The fact that seven (out of 192) employees use the car as a passenger shows that less than $4 \%$ of employees use the car-pooling for commuting.

Another analysis in the economic aspect is directly related to displacement. Fig. 13 shows the distribution of employees according to the monthly cost incurred for the ride. Just over half of the employees bears a cost of less than $€ 50$, more than a quarter spends between $€ 50$ and $€$ 100 , about $20 \%$ at a cost of more than $€ 100 /$ month, and some of these more than $150 € /$ month.

Exploding the graph of Fig. 13, Fig. 14 shows that the mode of transport car as driver is the only cost more than $€ 100$ /month for more than $20 \%$ of employees who have chosen it. In addition, the car-pooling allows more than $80 \%$ of employees who use it to keep costs below $50 €$.
As the private car is the means of transport most widely used by the employees, we have analysed the reasons for this choice. $92 \%$ of the reasons lies in the aspects that affect the need to minimize the time spent traveling to and from work (excessive travel time, lack of direct connections, occasional connections, stops away); only $2 \%$ believed that the use of public transport is not an economic alternative.

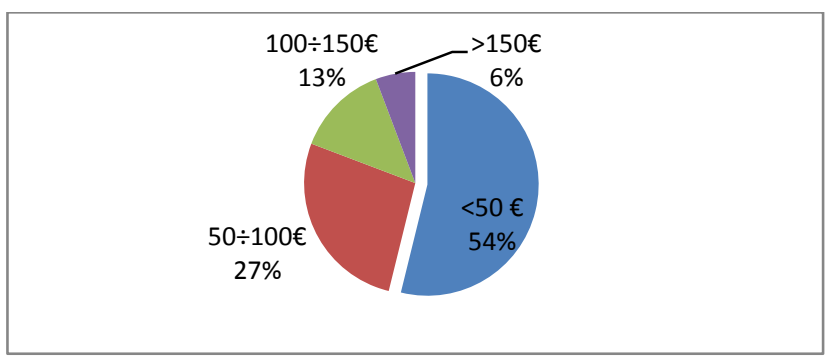

Fig. 13. Monthly cost incurred by the employees of the City of Andria for the home-work

Given the previous result, it is asked to employees under what conditions they would be willing to use public transport. The results (Fig. 15) highlight the critical issues related to the ability to reach the workplace in short times $(15 \%)$ and at convenient times $(19 \%)$. $19 \%$ of employees would not use anyway public transport; a full $41 \%$ did not give any response. Areas for improvement are clearly identifiable in changing times and in improving link services (about 34\%).

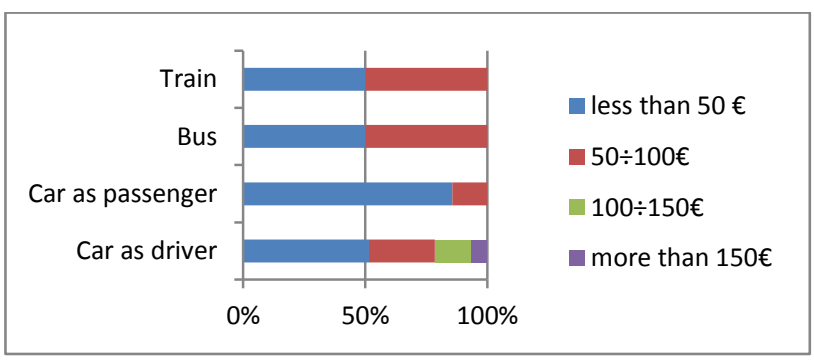

Fig. 14. Distribution of transport modes as a function of the costs incurred by the employees of the City of Andria

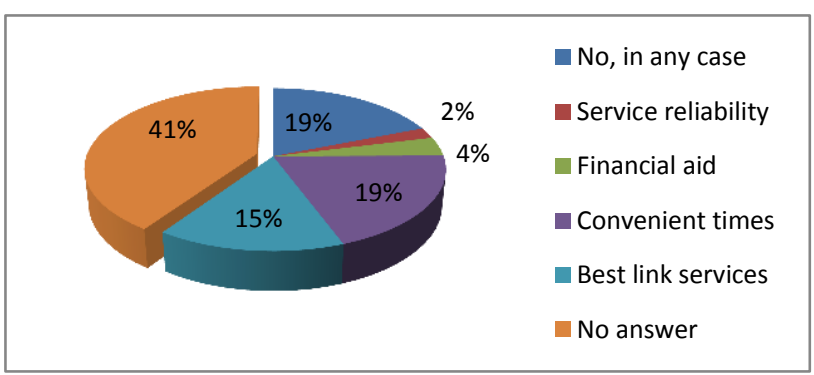

Fig. 15. Conditions of the employees of the City of Andria to use public transport

Other than public transport have been also evaluated (Fig. 16). Car-pooling (already used by some employees) is evaluated as a possible alternative by $37 \%$ of employees who use the car, provided that this does not result in a dilation of the travel time. The bike-sharing is considered as a possible alternative by $53 \%$ of employees self-equipped (Fig. 17), provided that there are stalls equipped bike paths and bike hire public. The remaining $47 \%$ does not evaluate this possibility in any case. 


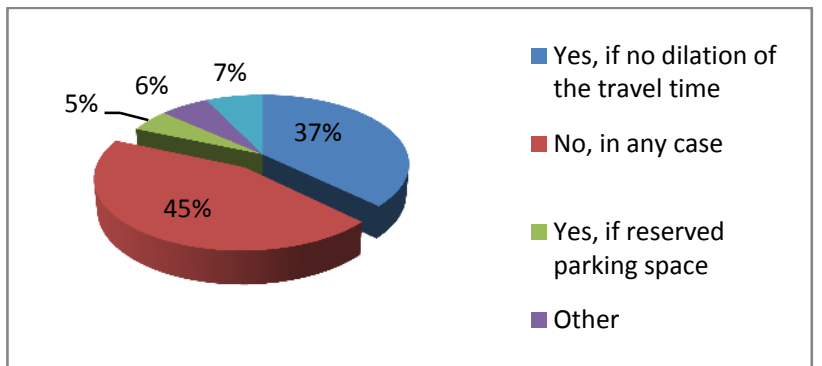

Fig. 16. Conditions of the employees of the City of Andria to use the car-pooling

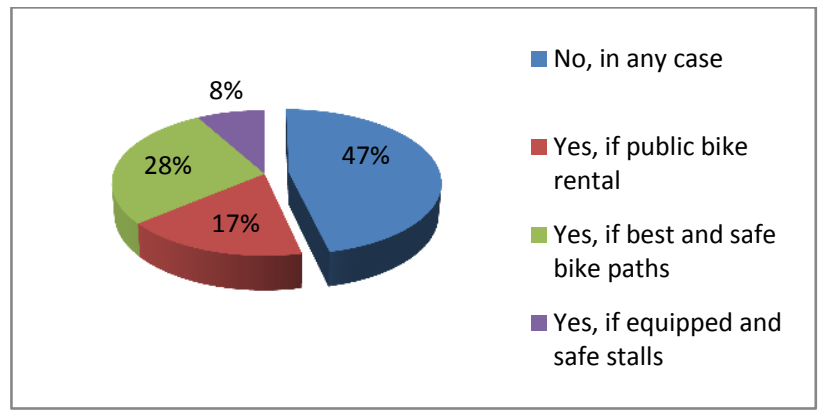

Fig. 17. Conditions of the employees of the City of Andria to use the bike-sharing

\section{Municipality of Trani}

The number of municipal employees is 135 , of which 49 $(36 \%)$ was not available to respond to the questionnaire. The completed questionnaires were 86 (64\%), of which 73 belong to resident employees ( $84 \%$ of the dataset).

The first analysis concerns the mode of travel from home to work. Fig. 18 shows the number of employees for each type, which shows that the private car is the main means of transport, followed by mode afoot. Very low is the use of public transport, especially the train (typically used by staff outside-seat), being very low number of employees off-seat. The use of the private car (driver + passenger) represents about $60 \%$ of the mode of travel. This, probably, takes into account the fact that as many as 45 employees have a private parking space in their house, which, in addition to being a head start from home, avoiding the problem of parking to return from work; Furthermore, all employees say they benefit from free parking, when they reach the workplace. The sum of all the values do not reach the number of questionnaires (86) as some employees did not give any indication of the means of transport.

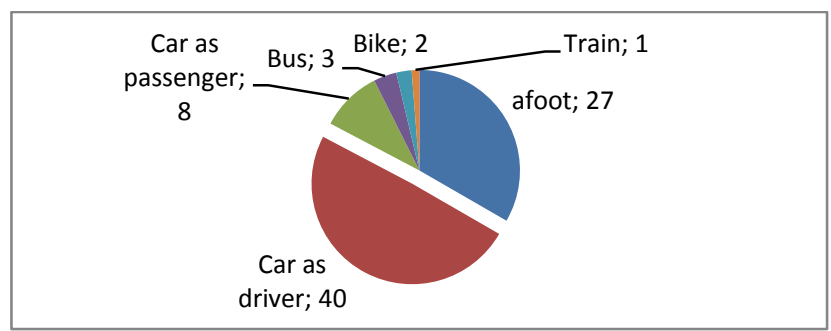

Fig. 18. Modes of travel to and from work for employees of the City of Trani

Another analysis concerned the time needed to reach the bus transportation mode closest to your home. Fig. 19 shows the time needed to reach the nearest bus stop, taking into account the mobility mode currently used.

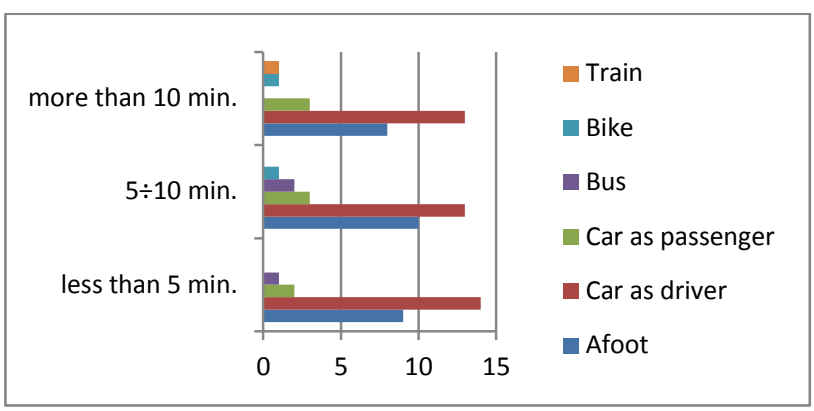

Fig. 19. Time to reach the stop nearest to your home for the employees of the City of Trani

It appears that the private car is the most widely used regardless of the time required to reach the stop in an alternate means. Even the bycicle is used to route the average.

Similarly, it appears that the income of the employee does not influence the decision on the means of transport to and from work. In fact, the private car has been majority used by employees with average income as well as by employees with low income, with distributions similar to those of the other two cities. Finally only 17 employees out of 86 say they have to do in the intermediate displacements from work, typically to accompany (or resume) children in sole or assistance to elderly or disabled parents or commissions. All 17 employees use your own car. Whereas the private car is used by 40 employees, this implies that 23 employees (plus 8 as passengers) use this medium to the only way to work, regardless of the distance and the time required to travel the distance. The fact that 7 (of 48) employees use the car as a passenger showed a $14 \%$ of employees who use the car-pooling for commuting, higher than the other two cities survey. Another analysis in the economic aspect directly related to displacement. Fig. 20 shows the distribution of employees according to the monthly cost incurred for the ride. Almost two-thirds of the employees support a cost of less than $€ 50$, while about a third spend between $€ 50$ and $€ 100$; only $4 \%$ at a cost of more than $€$ $100 /$ month.

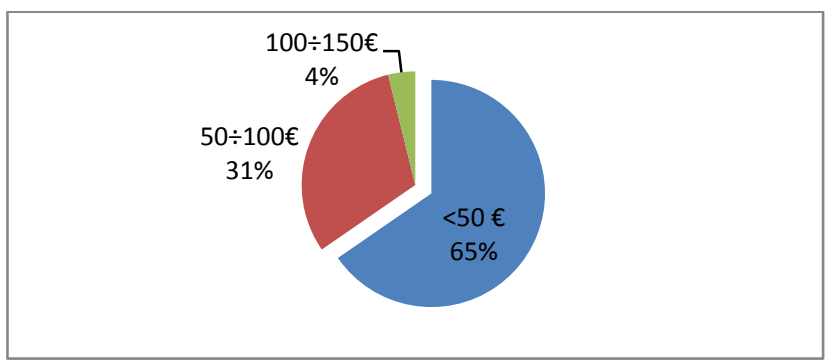

Fig. 20. Monthly cost incurred by the employees of the City of Trani for the home-work

Exploding the graph of Fig. 20, Fig. 21 shows that the mode of transport car as driver has the smallest percentage of employees (just over $60 \%$ ) with a monthly cost of less than $50 €$; then as much as $40 \%$ of employees who use their own car has a monthly cost above $50 €$ and a $4 \%$ cost in excess of $€ 100$. 


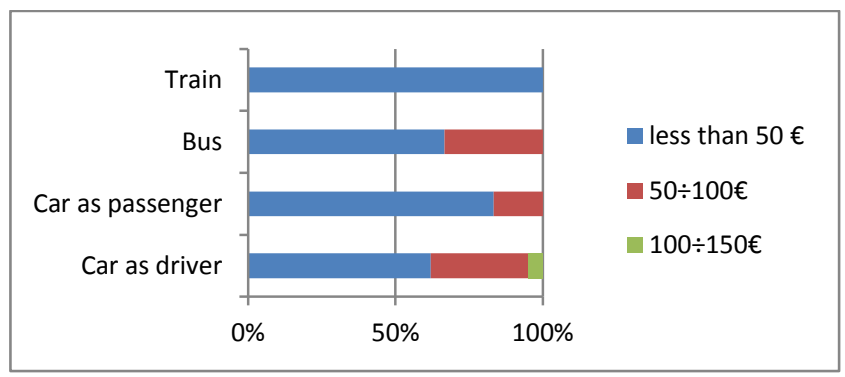

Fig. 21. Distribution of transport modes as a function of the costs incurred by the employees of the City of Trani

As the private car is the means of transport most widely used by the employees, we have analysed the reasons for this choice. $95 \%$ of employees identifies the causes specified, related to public transport. For example, $11 \%$ believed that the use of public transport is not a cheap alternative, while $75 \%$ of the reasons lies in the aspects that affect the need to minimize the time spent traveling to and from work (excessive travel time, lack of direct connections, occasional connections).

Given the previous result, it is asked to employees under what conditions they would be willing to use public transport (Fig. 22).

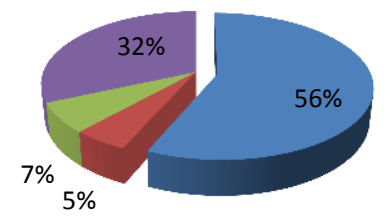

No, in any case

- Service reliability

Financial aid

- Convenient times

Fig. 22. Conditions of the employees of the City of Trani to use public transport

As many as $56 \%$ of them still would not use public transport, while $32 \%$ would consider if the times were adjusted to the needs of displacement; $7 \%$ would be willing to review the current choices in the presence of financial aid.

They have also evaluated other options. Car-pooling (Fig. 23 ) is evaluated as a possible alternative by $38 \%$ of employees who use the car, provided that this does not result in an expansion of the travel time. 13\% would consider it if there were reserved parking space, $14 \%$ already use it. The remaining $35 \%$ do not take into account it in any case. The bike-sharing (Fig. 24) is evaluated as a possible alternative by $55 \%$ of employees self-equipped, provided that there are stalls equipped (15\%), safe cycle tracks (25\%) and rental options or free use of bike (15\%), while the remaining $45 \%$ does not evaluate this possibility in any case.

\section{Conclusion}

This study has given several suggestions. First of all, the use of a private car cannot be easily changed, because of consolidated habits or intermediate stops. The medium used is not dependent from the income neither from the distance with respect to the nearest stop of a public medium. Moreover, it results that sometimes the reasons are related to the organization of the urban public transportation, whereas sometimes they depend from personal reasons. Alternative modes of mobility (as carpooling or bike-sharing) could be chosen by about half of the employees, if specific conditions were satisfied. Unfortunately, these conditions cannot be always satisfied. For these last cases, an effective solutions to mitigate the pollution could be the use of the electric vehicles, nevertheless these cities are not yet equipped with public electric charging stations which could reduce the $\mathrm{CO}_{2}$ emissions.

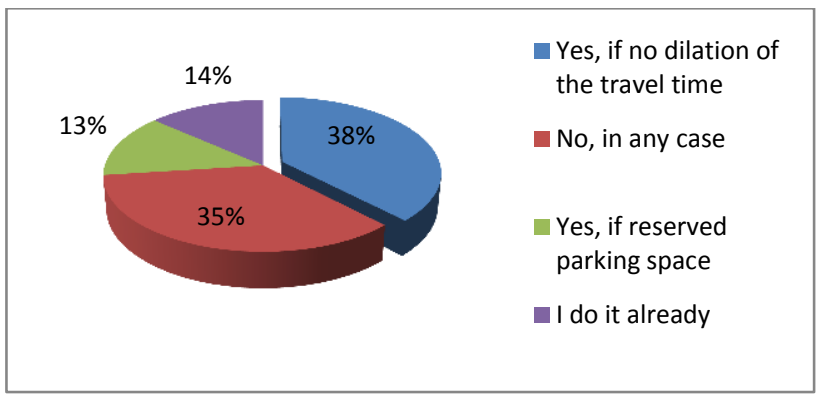

Fig. 23. Conditions of the employees of the City of Trani to use the car-pooling

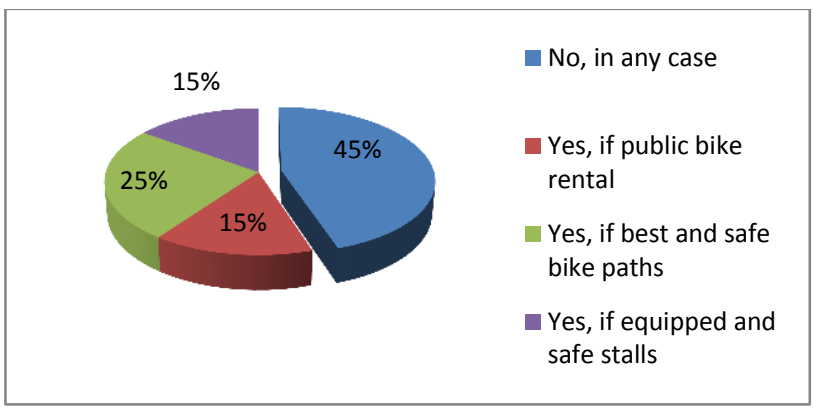

Fig. 24. Conditions of the employees of the City of Trani to use the bike-sharing

\section{Acknowledgement}

The paper represents an activity of a project funded by the BAT (Barletta-Andria-Trani) Province and it is also within the framework of the project "RES NOVAE Reti, Edifici, Strade - Nuovi Obiettivi Virtuosi per l'Ambiente e l'Energia". This project is supported by the Italian University and Research National Ministry (MIUR) and competitiveness program that Italy is developing to promote "Smart Cities Communities and Social Innovation".

\section{References}

[1] D. Schrank and T. Lomax, "2011 Annual urban mobility report," Texas Transp. Inst., College Station, TX, USA, Tech. Rep. 10-09-019, Sep. 2011.

[2] "Keep Europe moving - Sustainable mobility for our continent," European Commission, Brussels, Belgium, Tech. Rep. COM/2006/0314, Jun. 2006.

[3] "Towards a resource-efficient transport system," Copenhagen, Denmark, 2010.

[4] "Inventory of U.S. Greenhouse gas emissions and sinks: 1990-2010," Washington, DC, USA, Tech. Rep. EPA 430-R-12-001, 2012.

[5] "EU energy and transport in figures-Statistical pocketbook 2010," Brussels, Belgium, 2010.

[6] d'Orey P.M., Ferreira M., "ITS for Sustainable Mobility: Survey on Applications and Impact Assessment Tools", IEEE Trans. on Intelligent Transportation Systems, vol. 15 , no. 2, april 2014, pp. 477-493. 